\title{
Strategies to Investigate Grain Boundary Structure and Properties of Bulk Thermoelectric Samples
}

\author{
Jann A. Grovogui ${ }^{1}$, Poya Yasaei $^{1}$, Ian T. Witting ${ }^{1}$, G. Jeffery Snyder ${ }^{1}$ and Vinayak P. Dravid ${ }^{1 *}$ \\ ${ }^{1}$ Department of Materials Science and Engineering, Northwestern University, Evanston, IL, USA. \\ * Corresponding Author: v-dravid@northwestern.edu
}

In the effort to reduce carbon emissions, scientists and engineers have aimed to develop new energy generation and storage technologies. However, an equally important strategy is to decrease carbon emissions by using less resources. It is known that approximately $60 \%$ of the energy consumed globally is lost as wasted heat [1], yet some of this wasted heat can be converted into usable energy through the utilization of thermoelectric electricity generation. Currently, thermoelectric devices suffer from low efficiencies that originate in the innate coupling of competing electrical and thermal material properties. In order to optimize performance, electrical and thermal properties need to be decoupled, leading researchers to introduce microstructures into thermoelectric materials that impede heat flow yet allow electrons to move through the sample unimpeded.

Several computational works have investigated grain boundary (GB) properties in thermoelectric materials, yielding a variety of results that confirm the complexity of these defects. Novel boundary behaviours range from varying transmission coefficients for phonons of different wavelength, to extra electronic states appearing due to boundary twinning [2,3]. In addition, studies of 2D semiconducting materials have attempted to characterize GB properties as they relate to boundary structure [4]. However, commercially viable thermoelectrics are composed of $3 \mathrm{D}$ bulk materials, and therefore experimental investigations into the properties of bulk grain boundaries are critical to improving the performance of real-world devices.

Here, we devise a series of experiments that will enable us to both identify the relationship between specific boundaries and transport properties in real, bulk thermoelectric materials. Grain boundaries are inherently complex and contain 5 macroscopic Degrees of Freedom (DOF): 3 DOF attributed to the misorientation between the two grains that create the boundary, and 2 DOF defined by the orientation of the boundary in relation to the two grains. Transmission Electron Microscopy (TEM) is a superb technique for identifying boundary structure and chemistry, yet the images captured using this technique are 2D projections. Therefore, TEM will fail to capture all the degrees of freedom necessary to properly associate the structures observed with a specific boundary classification. To properly define the boundary, we use a combination of electron backscattered diffraction (EBSD) and Scanning/Transmission Electron Microscopy (S/TEM) to isolate and identify the degrees of freedom. These techniques can be combined with analytical microscopy, and microfabrication techniques to draw specific conclusions about the relationship between GBs and electrical and thermal transport properties. Utilizing this variety of techniques introduces additional sample preparation challenges due to specifications related to sample size and geometry. Consequently, we will discuss the sample preparation necessary to obtain samples (such as those seen in Figure 1 and 2.) that can be measured and characterized, while also discussing the workflow required to ensure that sample properties are measured without impeding the structural and chemical analysis of grain boundaries [5]. 
References:

[1] U.S. DOE. Quadrennial Technology Review: An Assessment of Energy Technologies and Research Opportunities (2015) p.1.

[2] Z Zheng et al., J. Appl. Phys. 116 (2014).

[3] KC Kim et al., Nat. Commun. 7 (2016), p. 1.

[4] H Li et al., Phys. Chem. Chem. Phys. 17 (2015), p. 11150.

[5] This work made use of the MatCI Facility which receives support from the MRSEC Program (NSF DMR-1720139) of the Materials Research Center at Northwestern University In addition, this work made use of the EPIC, facility of Northwestern University's NUANCE Center, which has received support from the Soft and Hybrid Nanotechnology Experimental (SHyNE) Resource (NSF ECCS1542205); the MRSEC program (NSF DMR-1121262) at the Materials Research Center; the International Institute for Nanotechnology (IIN); the Keck Foundation; and the State of Illinois, through the IIN. This work was supported by the U.S. Department of Energy, Office of Science, Office of Basic Energy Sciences, under Contract No. DE- SC0014520. This material is based upon work supported by the National Science Foundation Graduate Research Fellowship under Grant No. DGE-1324585.

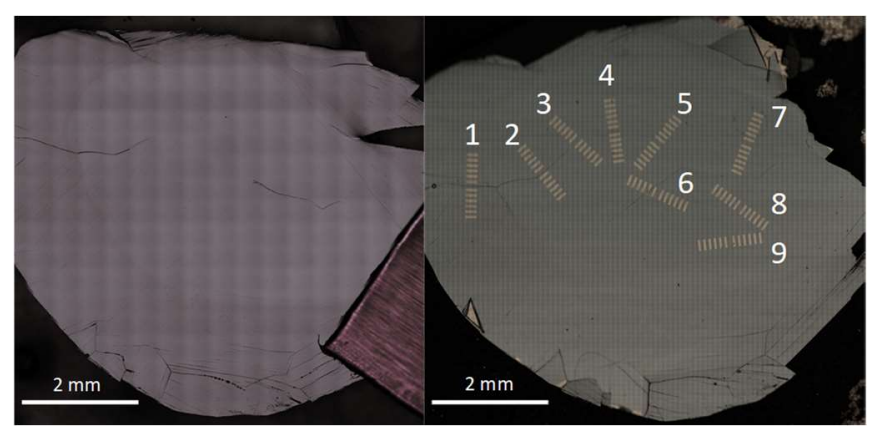

Figure 1. Optical montage images of a $50 \mu \mathrm{m}$ thick Bi2Te3 thermoelectric (left) before and (right) after $\mathrm{Au}$ contacts were deposited across 9 different grain boundaries. These boundaries were located using EBSD.

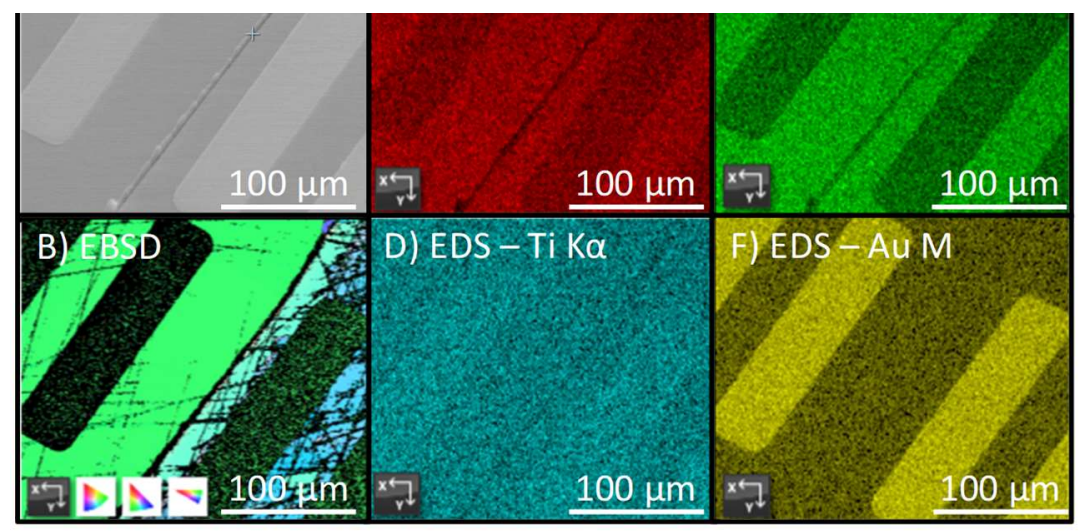

Figure 2. A) Secondary Electron image of electrodes patterned across a grain boundary and B) the electron backscattered diffraction (EBSD) map showing the change in grain orientation between metal electrode contacts. C-F) Energy Dispersive Spectroscopy (EDS) of the same area revealing the $\mathrm{Au}$ contacts are resting on either side of $\mathrm{a} \mathrm{Bi}_{2} \mathrm{Te}_{3}$ grain boundary, with no composition difference between the two grains. 